\title{
Protective effect of serotonin derivatives on glucose-induced damage in PC12 rat pheochromocytoma cells
}

\author{
Rosaria Piga $^{1}$, Yuji Naito ${ }^{2}$, Satoshi Kokura ${ }^{3}$, Osamu Handa ${ }^{3}$ and Toshikazu Yoshikawa ${ }^{1}$ \\ ${ }^{1}$ Department of Inflammation and Immunology, Graduate School of Medical Science, Kyoto Prefectural University of Medicine, \\ Kamigyo-ku, Kyoto, Japan \\ ${ }^{2}$ Department of Medical Proteomic, Graduate School of Medical Science, Kyoto Prefectural University of Medicine, Kamigyo-ku, \\ Kyoto, Japan \\ ${ }^{3}$ Department of Biomedical Safety Science, Graduate School of Medical Science, Kyoto Prefectural University of Medicine, \\ Kamigyo-ku, Kyoto, Japan \\ (Received 24 November 2008 - Revised 15 June 2009 - Accepted 17 June 2009 - First published online 14 September 2009)
}

Oxidative damage is believed to be associated with ageing, cancer and several degenerative diseases. Previous reports have shown that safflowerseed extract and its major antioxidant constituents, serotonin hydroxycinnamic amides, possess a powerful free radical-scavenging and antioxidative activity, paying particular attention to atherosclerotic reactive oxygen species (ROS)-related dysfunctions. In the present report, we examined a still unknown cell-based mechanism of serotonin derivatives against ROS-related neuronal damage, phenomena that represent a crucial event in neurodegenerative diseases. Serotonin derivatives $N$-( $p$-coumaroyl)serotonin and $N$-feruloylserotonin exerted a protective effect on high glucose-induced cell death, inhibited the activation of caspase- 3 which represents the last and crucial step within the cascade of events leading to apoptosis, and inhibited the overproduction of the mitochondrial superoxide, which represents the most dangerous radical produced by hyperglycaemia, by acting as scavengers of the superoxide radical. In addition, serotonin derivative concentration inside the cells and inside the mitochondria was increased in a time-dependent manner. Since recent studies support the assertion that mitochondrial dysfunctions related to oxidative damage are the major contributors to neurodegenerative diseases, these preliminary cell-based results identify a mitochondria-targeted antioxidant property of serotonin derivatives that could represent a novel therapeutic approach against the neuronal disorders and complications related to ROS.

Serotonin derivatives: Superoxide: Antioxidants: Apoptosis

Hyperglycaemia is associated with the progression of diabetic neuropathy caused by an insufficiency of arterial blood flow due to hyperglycaemia-induced atherosclerosis ${ }^{(1,2)}$, suggesting that hyperglycaemia may also damage neuronal cells because of the increased oxidative stress associated with disease progression $^{(3,4)}$. Mitochondria are a major source of intracellular reactive oxygen species (ROS) and are vulnerable to oxidative stress $^{(5)}$; their dysfunction leads to cell death via apoptosis and/or necrosis, playing a crucial role in the majority of neurodegenerative diseases ${ }^{(4,5)}$. Synthetic and natural antioxidants are widely used against oxidative damage as food additives and for tissue protection; because of the potential carcinogenic activity of the synthetic molecules, natural antioxidants are desirable $^{(6)}$. Many epidemiological and clinical studies have suggested that antioxidants play a preventive role in several oxidative stress-related diseases; however, many large-scale intervention trials have failed to demonstrate the beneficial effects of antioxidant vitamins ${ }^{(7)}$. Recently, some independent laboratories have focused their attention on other dietary compounds, such as serotonin derivatives, which may exert significant antioxidant effects both in vitro and in vivo, concentrating on their anti-atherogenic properties.

$N$-( $p$-coumaroyl)serotonin (CS) and $N$-feruloylserotonin (FS), two serotonin conjugates, have been identified as the major phenolic constituents of defatted safflower seeds ${ }^{(8,9)}$, which are members of the indole hydroxycinnamic acid amides, with serotonin (5-HT), p-coumaric acid ( $p$-ca) and ferulic acid (fa) representing components of their structures $^{(10,11)}$. The hydroxycinnamic acid amides of serotonin, including CS and FS, are synthesised by the enzyme serotonin $N$-hydroxycinnamoyltransferase.

CS and FS are present in several wild-growing plants and their seeds, such as safflower (Carthamus tinctorius L.) used in herbal medicine in Eastern countries, and worldwide mainly for edible oil production ${ }^{(9,10)}$, konnyaku (Amorphophallus konjac K. Koch) widely used as a traditional food all over Japan ${ }^{(12)}$, Japanese barnyard millet (Echinochloa utilis Ohwi \& Yabuno), widely used in Japan especially in cold districts because of its resistance to low temperatures and for its highly nutritious grains making it a good substitute

Abbreviations: CS, $N$-( $p$-coumaroyl)serotonin; fa, ferulic acid; FS, $N$-feruloylserotonin; HG, 50-mM-D-(+)-glucose (high glucose); 5-HT, serotonin; $p$-ca, $p$-coumaric acid; PI, propidium iodide; $p \mathrm{NA}$, $p$-nitroanilide; ROS, reactive oxygen species.

* Corresponding author: Dr Rosaria Piga, fax +81 75252 3721, email rosariapiga@tiscali.it 
for rice ${ }^{(13)}$, Centaurea nigra L. and many species of the genus Centaurea, traditionally used for their antibacterial activity in the treatment of various foods ${ }^{(14-16)}$, and maize bran and other grains used in a wide variety of ways, including home cooking, to increase the fibre content of various foods ${ }^{(17)}$. Furthermore, their presence has also been detected in several vegetables $^{(10)}$ used widely around the world.

Some of their biological and chemical activities have already been reported; they possess antibacterial and free radical-scavenging activities ${ }^{(12-14,17)}$, behave as antioxidants in plasma and on liver HDL-cholesterol and total cholesterol ${ }^{(18)}$, suggested to increase proliferation of fibroblasts ${ }^{(19)}$, inhibit the production of pro-inflammatory cytokines ${ }^{(19)}$, inhibit LDL oxidation in apoE-deficient mice ${ }^{(8)}$, exert protection against the increase of ROS-dependent adhesion and migration molecules that represent the key events in the early stages of atherosclerogenesis ${ }^{(1,20)}$, and show other benefits against cardiovascular risk in healthy human volunteers ${ }^{(21)}$. However, there are no reports ascribing a particular effect to these serotonin derivatives against high glucose-inducing neuronal damage, although several reported evidence of the involvement of high glucose on mitochondrial dysfunction and oxidative stress in neurodegenerative diseases ${ }^{(5,22,23)}$.

The PC12 cell line, derived from a rat pheochromocytoma of adrenal medulla, undergoes differentiation following treatment with nerve growth factor from a tumour cell (tumour-type) to a neuron-like cell phenotype (neurontype $)^{(24,25)}$. The neuron-type cell is known to express a variety of properties shared by neurons including the overall phenotype and has been successfully used over the years as a neuronal model to study neuronal functions ${ }^{(26-29)}$.

The present study followed a cell-based approach to investigate the protective effect of CS and FS against damage associated with hyperglycaemia in PC12 cells.

\section{Materials and methods}

\section{Cells}

Undifferentiated PC12 cells (kindly provided by Dr Hirose, Department of Anaesthesiology, Kyoto Prefectural University of Medicine, Kyoto, Japan) were routinely maintained at $37^{\circ} \mathrm{C}$ in a humidified atmosphere of $95 \%$ air and $5 \% \quad \mathrm{CO}_{2}$ in Dulbecco's modified Eagle's medium-Ham's nutrient mixture F-12 (DMEM-F-12, 1:1; Invitrogen, Carlsbad, CA, USA), containing $17.5 \mathrm{~mm}-\mathrm{D}-(+)$-glucose, $10 \%$ heatinactivated fetal bovine serum and $5 \%$ heat-inactivated horse serum. At $60 \%$ of confluence, the cells were differentiated by the administration of nerve growth factor $(100 \mathrm{ng} / \mathrm{ml})$ to a DMEM-F-12 medium supplemented with $0.5 \%$ fetal bovine serum and $0.5 \%$ horse serum for $4 \mathrm{~d}$, as previously described ${ }^{(30)}$. Differentiated PC12 cells without any treatment and growing in DMEM-F-12 medium containing sufficient $\mathrm{D}-(+)$-glucose were used as control cells.

\section{Cell treatment}

Differentiated PC12 cells were treated with $\mathrm{D}-(+)$-glucose at different concentrations $(25,50,100 \mathrm{mmol} / \mathrm{l})$ for specified periods $(0,24,48,72,96 \mathrm{~h})$ as well as with 50 -mm-D-(+)glucose (high glucose; $\mathrm{HG}$ ) for $72 \mathrm{~h}$, which represent the resulting $50 \%$ lethal concentration $\left(\mathrm{LC}_{50}\right)$, in the presence or absence of $10 \mu \mathrm{M}-\mathrm{CS}$ or -FS (kindly provided by Dr Koyama, Ajinomoto Co., Kawasaki, Japan), $10 \mu \mathrm{M}-5-\mathrm{HT}$, - $p$-ca or -fa as the components of CS and FS structures or $40 \mu \mathrm{M}$ - $\alpha$-tocopherol which was used as a positive control (all from Sigma, St Louis, MO, USA). Glucose uptake was performed as previously described ${ }^{(31)}$ using a fluorescent D-glucose analogue 2-[N-(7-nitrobenx-2-oxa-1,3-diazol-4-yl) amino]-2-deoxy-D-glucose (Invitrogen), which was used to monitor glucose uptake in living cells.

\section{Cell viability}

To evaluate cell viability, the 3-(4,5-dimethylthiazol-2-yl)-2, 5-diphenyltetrazolium bromide (Sigma) assay was performed as described previously ${ }^{(32)}$. The optical density of formazan was measured at $570 \mathrm{~nm}$ using a Multiskan Ascent plate reader (Bio-Rad Fluoromark, Melville, NY, USA).

Phosphatidylserine, which is confined to the inner membrane in normal healthy cells, becomes exposed at the outer plasma membrane of apoptotic cells ${ }^{(33)}$. Exposure of phosphatidylserine is an early event, as it precedes cell membrane permeabilisation, cell shrinkage and nuclear condensation $^{(33)}$. Phosphatidylserine exposure was then analysed as described previously ${ }^{(33)}$, and measured by the binding of annexin V-zFITC, according to the protocol outlined by the manufacturer of the Annexin V-FITC Apoptosis Detection kit (MBL, Nagoya, Japan). Cells that have lost membrane integrity allow propidium iodide (PI) to enter. Treated cells were also stained with PI (MBL, Nagoya, Japan), and analysed using a Cytomics FC500 Flow Cytometry System.

\section{Caspase activity}

Caspase activity was measured by assessing the cleavage of the Asp-Glu-Val-Asp peptide-conjugated $p$-nitroanilide $(p N A)^{(34)}$ (Caspase-3/CPP32 Colorimetric Protease Assay Kit; MBL, Nagoya, Japan), according to the manufacturer's protocol. Substrate cleavage, which resulted in the release of $p$ NA (405 nm), was measured using a Multiskan Ascent plate reader (Bio-Rad). Absorbance units were converted to pmol $p$ NA by extrapolating them on a standard curve generated using free $p$ NA. Caspase activity was expressed as pmol $p$ NA generated per mg protein (Bio-Rad protein assay).

\section{Determination of intracellular reactive oxygen species}

Intracellular ROS were detected using the fluorescent probe $2^{\prime}, 7^{\prime}$-dichlorofluorescin diacetate (Molecular Probes, Eugene, OR, USA) as described earlier ${ }^{(35)}$. Mitochondrial superoxide was detected using a MitoSOX ${ }^{\mathrm{TM}}$ mitochondrial superoxide indicator (Molecular Probes). Fluorescence was observed using the Cytomics FC500 Flow Cytometry System.

\section{High-performance liquid chromatography analysis}

A sample of cells was used for mitochondria extraction performed using a Mitochondria Isolation Kit (Pierce, Rockford, IL, USA) according to the manufacturer's protocol. Subsequently, CS and FS were extracted with methanol-PBS $(4: 1, \quad \mathrm{v} / \mathrm{v})$ and were measured by HPLC as described 
earlier $^{(36)}$. In order to quantify the concentration, CS and FS levels were normalised by the total protein content ( $\mu \mathrm{mol} / \mathrm{mg}$ protein).

\section{Data analysis}

Statistical analysis was performed as described previously ${ }^{(37)}$. All data of at least three separate experiments were expressed as mean values with their standard errors. Inter-group differences were assessed by one-way ANOVA followed by the post hoc Bonferroni test. In order to assess the individual effects of HG and the selected compounds, as well as any interactions existing between these two factors, a two-way ANOVA with post hoc comparison was performed. All statistical analyses were carried out using the GraphPad Prism 4 program (GraphPad Software, Inc., San Diego, CA, USA). Values of $P<0.05$ were considered statistically significant.

\section{Results}

Effects of $\mathrm{N}-(\mathrm{p}$-coumaroyl)serotonin and $\mathrm{N}$-feruloylserotonin on 50-mM-D-(+)-glucose-induced damage in PC12 rat pheochromocytoma cells

D-(+)-Glucose treatment decreased PC12 viability in a concentration- and time-dependent manner and the resulting $50 \%$ lethal concentration $\left(\mathrm{LC}_{50}\right)$ was $50 \mathrm{mmol} / \mathrm{l}$ at $72 \mathrm{~h}$ (Fig. 1(a)). The viability decreased to approximately $24 \%$ $(P<0.05), 35 \%(P<0.01)$ and $44 \%(P<0.01)$ at 24,48 and $72 \mathrm{~h}$, respectively, at the concentration of $50 \mathrm{mmol} / \mathrm{l}$. Simultaneous treatment of PC12 cells with CS or FS plus $\mathrm{HG}$ for $72 \mathrm{~h}$ (Fig. 1(b)) resulted in a significant inhibition of cell death $(P<0 \cdot 001)$ in comparison with cells treated with $\alpha$-tocopherol $(P<0 \cdot 001)$, while treatment with $10 \mu \mathrm{M}-5-\mathrm{HT}$, $-p$-ca or -fa resulted in a significant but less protection $(P<0 \cdot 05)$. Cells were also incubated with annexin V-FITC and PI, and then subjected to flow cytometry analysis (Fig. 1(c)). The percentage of apoptotic cells (annexin V-FITC-positive and PI-negative cells) among the HG-treated cells was 14-fold higher than among control cells (from $2.7 \%$ of the controls to $37.9 \%$ of HG); CS and FS treatment was associated with a significant inhibition of apoptosis $(P<0 \cdot 01)$.

Effects of $\mathrm{N}-(\mathrm{p}$-coumaroyl)serotonin and $\mathrm{N}$-feruloylserotonin on 50-mM-D-(+)-glucose-induced caspase activity in PC12 rat pheochromocytoma cells

The caspase activity was analysed to confirm the ability of CS and FS to protect against apoptotic cell death caused by this stressor. As shown in Fig. 2(a), treatment of PC12 cells with HG caused a significant but transient increase in the caspase activity from 6 to $48 \mathrm{~h}$, being significant at $24 \mathrm{~h}(P<0 \cdot 01)$ and $48 \mathrm{~h}(P<0 \cdot 05)$. Caspase activity increased and decreased in a time-dependent manner and the resulting highest activity was approximately two-fold higher $(34.4 \mathrm{pmol} \mathrm{pNA} / \mathrm{mg}$ protein) than for control cells $(16.3 \mathrm{pmol} \mathrm{pNA} / \mathrm{mg}$ protein) at $24 \mathrm{~h}$. Thus CS and FS protection against caspase activity increase was analysed at $24 \mathrm{~h}$. Simultaneous treatment of PC12 cells with CS or FS plus HG for 24h (Fig. 2(b)) resulted in a significant inhibition of caspase activity $(P<0 \cdot 01)$, stronger than that exerted by $\alpha$-tocopherol $(P<0 \cdot 05)$.
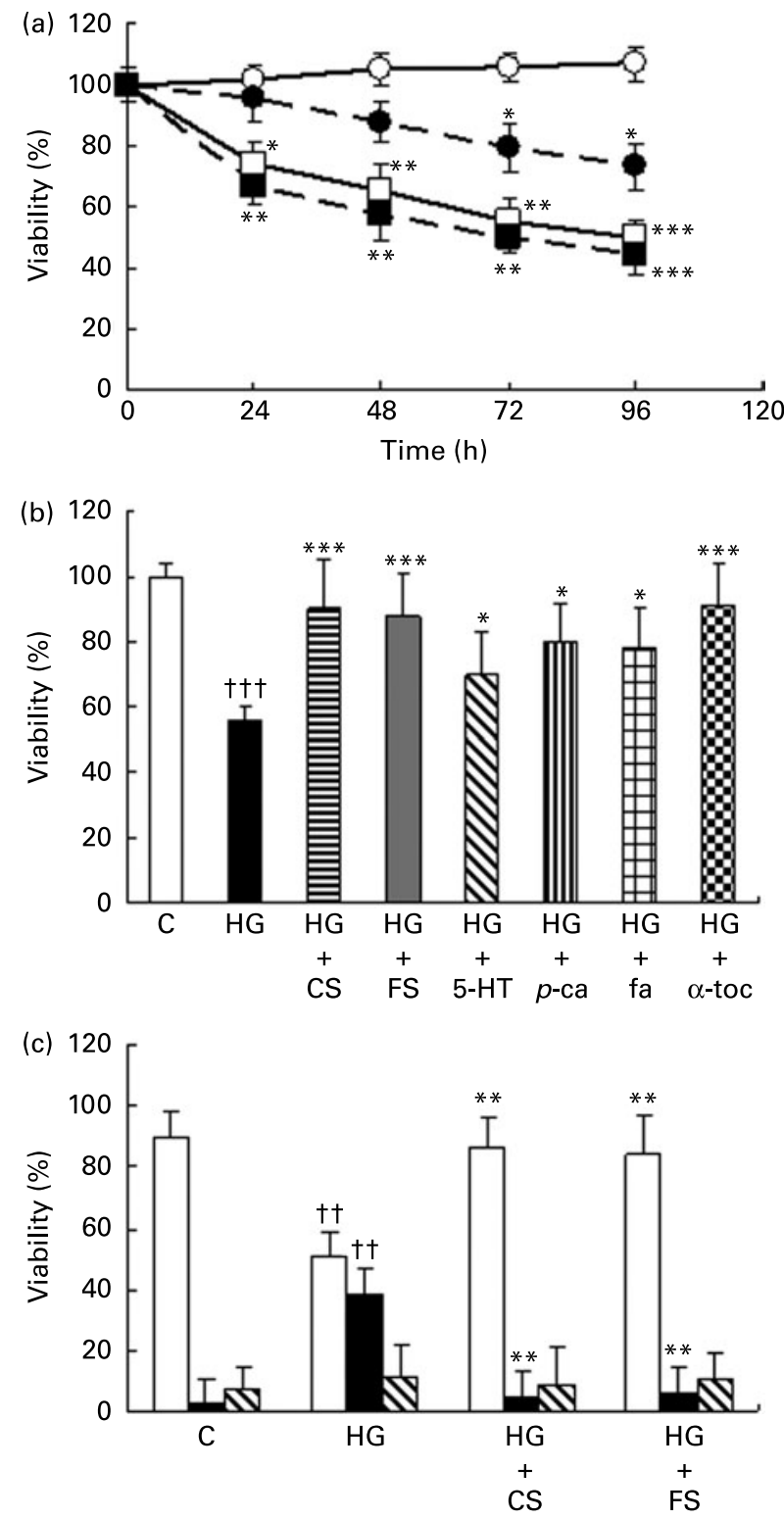

Fig. 1. Effects of $10 \mu \mathrm{M}-N-(p$-coumaroyl)serotonin (CS) and $10 \mu \mathrm{M}-N$-feruloylserotonin (FS) on 50-mM-D-(+)-glucose (HG)-induced damage in PC12 rat pheochromocytoma cells. (a) Concentration- and time-dependent viability. (-O-), Control (cells without any HG treatment); (- - - -), $25 \mathrm{mM}-\mathrm{HG}$; (- $\square-$ ), $50 \mathrm{~mm}-\mathrm{HG}$; (-- - ), $100 \mathrm{~mm}-\mathrm{HG}$. Values are means, with their standard errors represented by vertical bars. Mean value was significantly different from that of the control treatment at the same time point: ${ }^{\star} P<0.05$, ${ }^{\star \star} P<0.01,{ }^{\star \star \star} P<0.001$. (b) Effect of the selected compounds on HG-inducing cell death. $\mathrm{C}$, control treatment; 5-HT, $10 \mu \mathrm{M}$-serotonin; $p$-ca, $10 \mu \mathrm{M}-p$ coumaric acid; fa, $10 \mu \mathrm{M}$-ferulic acid; $\alpha$-toc, $40 \mu \mathrm{M}-\alpha$-tocopherol. Mean value was significantly different from that of the HG-alone treatment: ${ }^{*} P<0.05$, ${ }^{* * *} P<0.001$. $+\dagger \dagger M e a n$ value was significantly different from that of the control treatment $(P<0.001)$. (c) Effect of $\mathrm{CS}$ and $\mathrm{FS}$ on HG-inducing apoptosis. $(\square)$, Living cells; $(\square)$, apoptotic cells; $(\mathbb{\mathbb { Q }})$, necrotic cells. ${ }^{\star *}$ Mean value was significantly different from that of the HG-alone treatment $(P<0.01)$. †† Mean value was significantly different from that of the control treatment $(P<0.01)$.

Effect of $\mathrm{N}-(\mathrm{p}$-coumaroyl)serotonin and $\mathrm{N}$-feruloylserotonin on 50-mM-D-(+)-glucose-induced intracellular reactive oxygen species

To understand the underlying mechanisms of CS and FS protection against HG damage, we examined the ROS-scavenging 

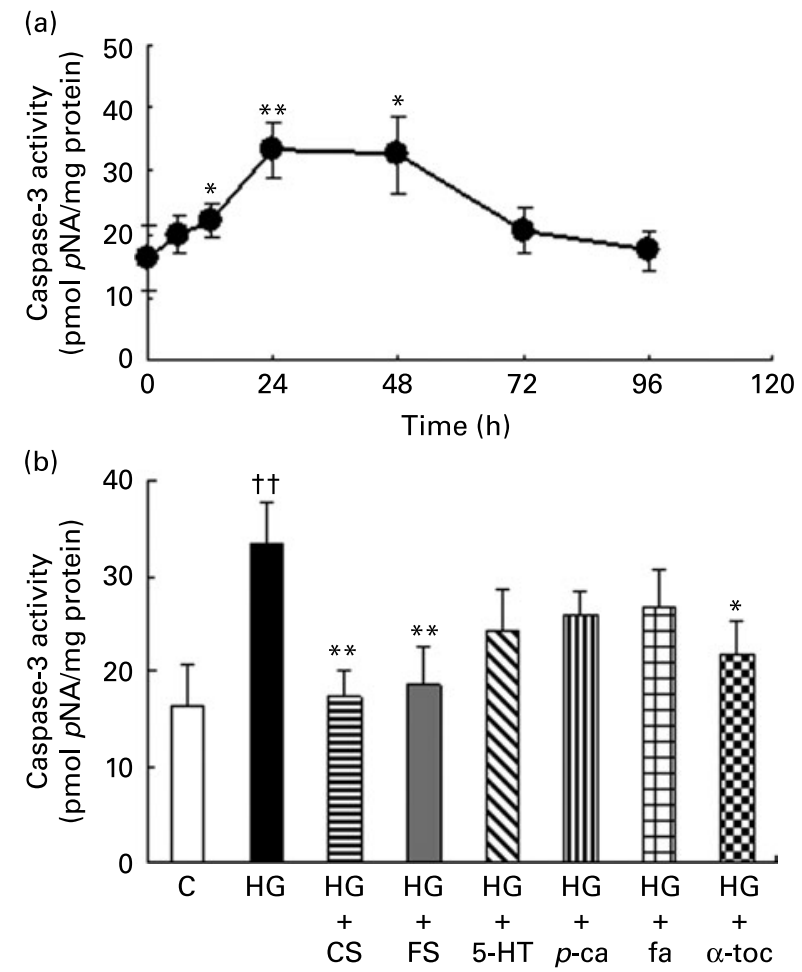

Fig. 2. Effects of $10 \mu \mathrm{M}-N-(p$-coumaroyl)serotonin (CS) and $10 \mu \mathrm{M}-N$-feruloylserotonin (FS) on 50-mm-D-(+)-glucose (HG)-induced caspase-3 activity in PC12 cells. Caspase-3 activity was measured by the cleavage of the Asp-Glu-Val-Asp (DEVD) peptide-conjugated $p$-nitroanilide $(p N A)$ and expressed per $\mathrm{mg}$ total protein content. (a) Effect of $\mathrm{HG}$ on caspase-3 activity. Values are means, with their standard errors represented by vertical bars. Mean value was significantly different from that at time 0 : ${ }^{*} P<0.05$, ${ }^{* *} P<0.01$. (b) Effect of the selected compounds on HG-inducing caspase activity. C, control (cells without any treatment); $5-\mathrm{HT}, 10 \mu \mathrm{M}$-serotonin; $p$-ca, $10 \mu \mathrm{M}$ - $p$-coumaric acid; fa, $10 \mu \mathrm{M}$-ferulic acid; $\alpha$-toc, $40 \mu \mathrm{M}-\alpha$-tocopherol. Mean value was significantly different from that of the HG-alone treatment: ${ }^{*} P<0.05,{ }^{* *} P<0.01$. †† Mean value was significantly different from that of the control treatment $(P<0.01)$.

activity of CS and FS. The HG-induced caspase activation on PC12 was preceded by a significant increase in the intracellular ROS levels $(P<0.01$ at $6,12,24 \mathrm{~h})$, as shown by the $2^{\prime}, 7^{\prime}$-dichlorofluorescin fluorescence (Fig. 3(a)). The peak of fluorescence was reached at $12 \mathrm{~h}$, although this increase was significant from $6 \mathrm{~h}$, and was significantly reduced by $\mathrm{CS}$ and FS $(P<0.01)$ (Fig. 3(b)). $40 \mu \mathrm{M}-\alpha$-Tocopherol had a similar effect to $10 \mu \mathrm{M}-\mathrm{CS}$ and $-\mathrm{FS}$, while treatment with $10 \mu \mathrm{M}$ $p$-ca or -fa resulted in a lower but still significant reduction of ROS fluorescence $(P<0.05)$ (Fig. 3(b)). A similar protocol was performed using a MitoSOX ${ }^{\mathrm{TM}}$ fluorescent probe, specific for mitochondrial superoxide. The four-fold increased fluorescence by $\mathrm{HG}$ at $6 \mathrm{~h}$ was significantly reduced by $\mathrm{CS}$ and FS $(P<0 \cdot 01)$ (Fig. 3(c) and (d)); $40 \mu \mathrm{M}-\alpha$-tocopherol resulted in a lower but still significant reduction of MitoSOX $^{\mathrm{TM}}$ fluorescence $(P<0 \cdot 05)$ (Fig. 3(c)).

\section{$\mathrm{N}-(\mathrm{p}$-coumaroyl)serotonin and $\mathrm{N}$-feruloylserotonin} concentration inside PC12 rat pheochromocytoma cells and mitochondria

Treatment with $20 \mu \mathrm{M}-\mathrm{CS}$ from 0 to $96 \mathrm{~h}$ resulted in a timedependent increase of CS concentration in cells and in
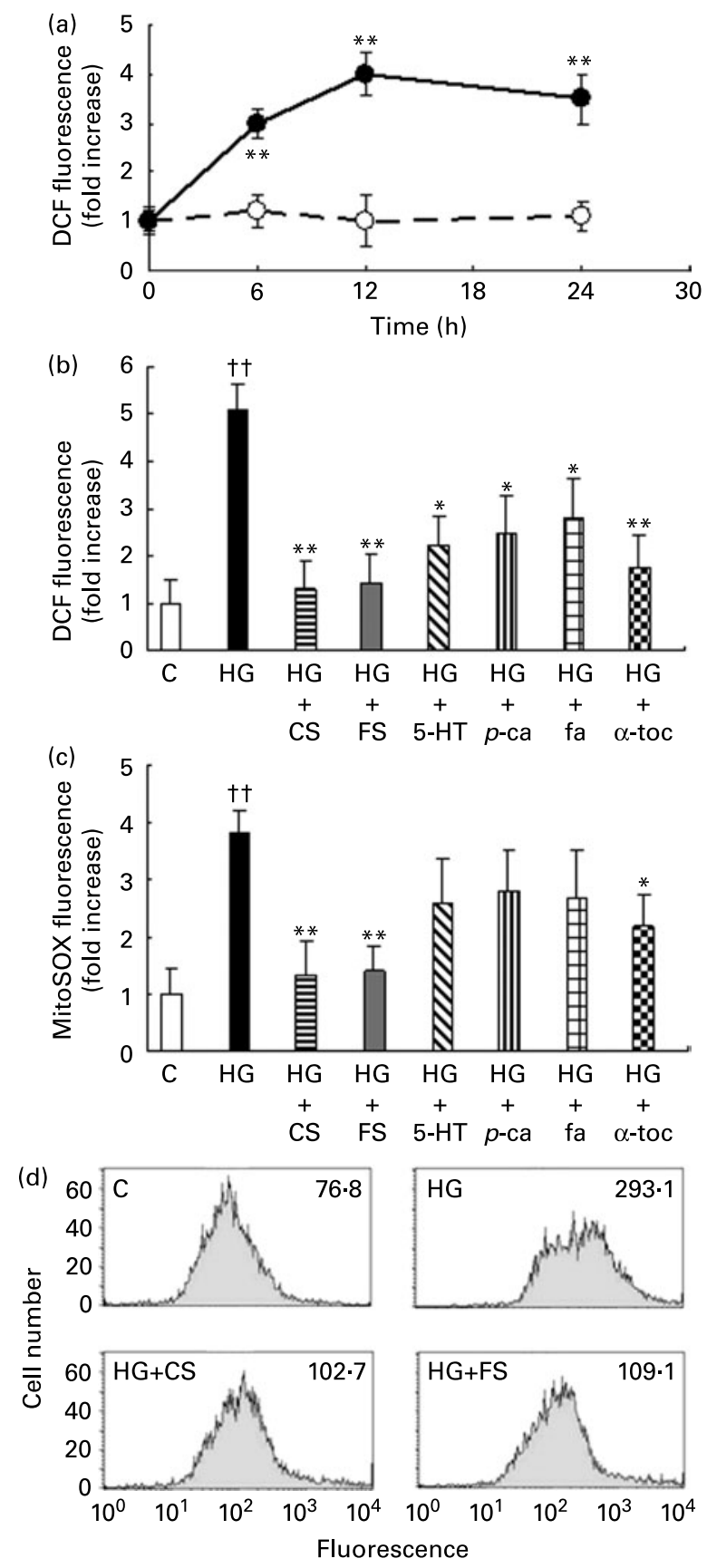

Fig. 3. Effect of $10 \mu \mathrm{M}-N-(p$-coumaroyl)serotonin (CS) and $10 \mu \mathrm{M}-N$-feruloylserotonin (FS) on 50-mM-D-(+)-glucose (HG)-induced intracellular reactive oxygen species (ROS) in PC12 cells. (a) Time-dependent intracellular ROS assessed by the fluorescence intensity of $2^{\prime}, 7^{\prime}$-dichlorofluorescin (DCF) emission in control (--O--) and HG-treated (- -) cells. Values are means, with their standard errors represented by vertical bars. ${ }^{* *}$ Mean value was significantly different from that at time $0(P<0.01)$. (b) Effect of the selected compounds on HG-inducing intracellular ROS assessed by the fluorescence intensity of DCF emission. C, control (cells without any treatment); 5- HT, $10 \mu \mathrm{M}$-serotonin; $p$-ca, $10 \mu \mathrm{M}$-coumaric acid; fa, $10 \mu \mathrm{M}$-ferulic acid; $\alpha$-toc, $40 \mu \mathrm{M}-\alpha$-tocopherol. Mean value was significantly different from that of the HG-alone treatment: ${ }^{\star} P<0.05,{ }^{\star \star} P<0.01$. †† Mean value was significantly different from that of the control treatment $(P<0.01)$. (c) Effect of the selected compounds on HG-inducing intracellular ROS assessed by the fluorescence intensity of MitoSOX ${ }^{\mathrm{TM}}$ emission. Mean value was significantly different from that of the HG-alone treatment: ${ }^{*} P<0.05$, ${ }^{* *} P<0.01$. †† Mean value was significantly different from that of the control treatment $(P<0.01)$. (d) Flow-cytometry images of MitoSOX ${ }^{\mathrm{TM}}$ fluorescence. 
mitochondria, reaching significance after $24 \mathrm{~h}$ in cells (10.4 $\mu \mathrm{mol} \mathrm{CS/mg} \mathrm{protein;} P<0.01)$, and in mitochondria (4.3 $\mu \mathrm{mol} \mathrm{CS/mg} \mathrm{protein;} P<0.05$ ) (Fig. 4(a)). A similar pattern was obtained for FS detection in cells $(9.6 \mu \mathrm{mol} \mathrm{FS} / \mathrm{mg}$ protein; $P<0.01)$ and in mitochondria $(2.9 \mu \mathrm{mol} \mathrm{FS} / \mathrm{mg}$ protein; $P<0.05$ ) (Fig. 4(b)).

\section{Discussion}

We previously reported that CS and FS, two serotonin conjugates, exerted their benefits against hyperglycaemic disorders and complications related to $\operatorname{ROS}^{(11)}$. Although increasing evidence suggests that $\mathrm{CS}$ and FS are protective against endothelial damage both in vitro and in vivo animals and human subjects ${ }^{(8,11,21)}$, to the best of our knowledge, cell-based studies on their action against neuronal damage have not been reported. Since mitochondrial dysfunction and oxidative stress are often correlated with hyperglycaemia $^{(5,22,23)}$, we questioned whether CS and FS have the potential to safely modulate oxidative stress in a neuronal system of hyperglycaemia toxicity, acting as protective compounds as they do in vascular systems.

In our cell-based neuronal-like system of hyperglycaemia toxicity, we found that the simultaneous treatment of PC12 with HG plus CS or FS reduced HG-induced cell death. Since the literature on CS and FS is scarce and in order to quantify their antioxidant capacity, we compared the effects of CS and FS with those of $\alpha$-tocopherol at a dietary relevant level $(40 \mu \mathrm{mol} / \mathrm{l})$. $\alpha$-Tocopherol is able to protect neuronal and PC12 cells from cell damage induced by several stresses including high glucose $\mathrm{e}^{(14,38,39)}$. Koyama et al. ${ }^{(21)}$ did not
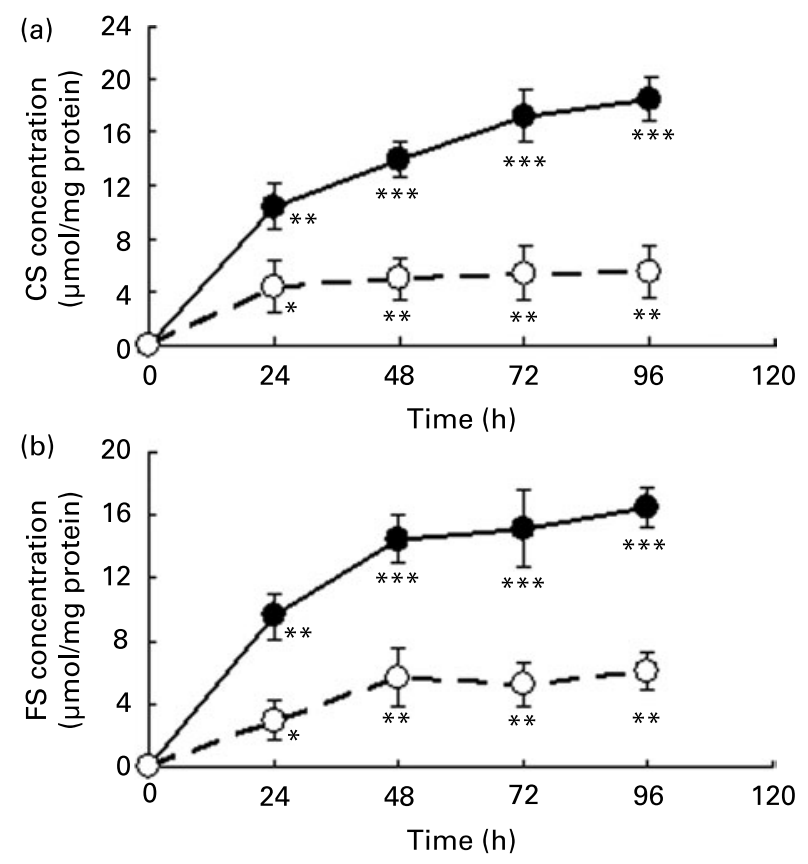

Fig. 4. $N$-(p-coumaroyl)serotonin (CS) (a) and $N$-feruloylserotonin (FS) (b) concentration inside PC12 whole cells (--) and mitochondria (--O- -). CS and FS levels were normalised by the total protein content $(\mu \mathrm{mol} / \mathrm{mg}$ protein). Values are means, with their standard errors represented by vertical bars. Mean value was significantly different from that at time 0 : ${ }^{\star} P<0.05$, ${ }^{\star \star} P<0.01,{ }^{\star \star \star} P<0.001$. measure directly the human plasma concentration of CS and FS, but they observed a whole-blood serotonin increase in association with CS or FS intake, while Tanno et al. ${ }^{(40)}$ measured a rapid absorption and relatively high blood concentrations of CS and FS in rats after oral administration. Although the present results are restricted to a cell-based approach, together with the evidence reported in the literature, it may be suggested that CS and FS are protective in our model and that levels necessary for this protection can be achieved through dietary means. We also compared the present results with the effect of 5-HT, $p$-ca or fa, which are the components of CS and FS structure, in order to show that the protection provided by FS and CS was greater than any component of their structure; CS and FS protection was higher than that of 5-HT, $p$-ca or fa alone in the experimental conditions used. Since 5-HT, $p$-ca and fa offer protection against cell death caused by stressors including glucose ${ }^{(38,39,41-44)}$, the present results suggest that protection offered by CS and FS may be due to both 5-HT and p-ca or fa moieties acting together. CS and FS protection was further demonstrated by using annexin V-FITC and PI fluorescent probes, allowing us to discriminate between apoptotic and necrotic cell death, underlining the ability of CS and FS to protect against apoptosis, which resulted as being the type of cell death resultant from HG. This result suggests that CS and FS may act in one or more of the cascade of events leading to apoptosis.

In order to understand the protective mechanisms against cytotoxicity and the effect on the apoptotic process, we examined the effect of CS and FS on caspase activity and ROS production. Hyperglycaemia may damage neuronal cells by increasing oxidative stress associated with a progression of diabetic neuropathy ${ }^{(3,4)}$. Mitochondria are the major endogenous producers of ROS, and they have been implicated in the control of cell death ${ }^{(45)}$. Mitochondria producing ROS could activate apoptotic cells death in two ways; through caspase-dependent and/or -independent pathways ${ }^{(45)}$. Caspase3 is required for the cleavage of the DNA fragmentation factor DFF, which has the nuclease activity responsible for DNA fragmentation during apoptosis ${ }^{(45)}$. Mitochondria, on the other hand, after apoptotic insult release apoptotic-inducing factor into the cytosol, which translocates to the nucleus where it binds to the DNA and induces its fragmentation, independently of caspase activity ${ }^{(45)}$. We tested the effect of CS and FS in comparison with the effects of $\alpha$-tocopherol, 5-HT, $p$-ca and fa on HG-induced caspase activity, to evaluate their role in PC12 cell death. The mitochondrial ROS distribution was also determined to clarify and compare their ability to penetrate inside mitochondria, and their role against superoxide, which is one of the most damaging radicals produced by hyperglycaemia ${ }^{(46)}$. The present results underline a role for caspase-3 in HG-inducing PC12 cell death; decreased activity of caspase- 3 and cell loss due to increased cells death. Both CS and FS inhibited caspase activity, being more protective than $\alpha$-tocopherol, which since none of the component structures affected caspase activity is probably associated with mitochondrial superoxide scavenging.

Although $2^{\prime}, 7^{\prime}$-dichlorofluorescin fluorescence was inhibited to different extents by each antioxidant used, only CS and FS possessed the ability to reduce the MitoSOX ${ }^{\mathrm{TM}}$ fluorescence an ability that $\alpha$-tocopherol also exerted with a similar 
fluorescence, being greater in treated than control cells. Since $2^{\prime}, 7^{\prime}$-dichlorofluorescin diacetate is not specific for superoxide, and does not penetrate selectively into mitochondria, this result means that superoxide is probably the chief radical produced by $\mathrm{HG}$, and it is localised inside the mitochondria. All the antioxidants tested scavenge cytosolic ROS other than superoxide, while only CS and FS scavenge mitochondrial superoxide. $\alpha$-Tocopherol, 5-HT, $p$-ca and fa activity against several ROS is already documented in the literature ${ }^{(38,39,41-44)}$. The present results, therefore, suggest a more powerful and selective antioxidant activity for CS and FS against mitochondrial superoxide which could be explained by a greater ability to cross the mitochondrial membrane. In fact, all the isoforms of vitamin $\mathrm{E}$ are lipophilic and are retained in cell membranes, failing to achieve significant intracellular concentrations as well at the relevant sites of free radical generation $^{(5)}$, while fa shows more pronounced mitigation of ROS in a combination with both ascorbic acid and vitamin $\mathrm{E}^{(44)}$. The benefits of $p$-ca under physiological conditions may be counteracted by its pro-oxidant activity ${ }^{(41,42)}$. These different actions with respect to scavenging superoxide could also explain their respective capacities to prevent cell death.

Since MitoSOX ${ }^{\mathrm{TM}}$ results confirmed an early uptake of CS and FS into the mitochondria, we quantified the concentration of both compounds inside the $\mathrm{PC} 12$ cells and their mitochondria by HPLC. Treatment of PC12 cells with CS or FS revealed a time-dependent increase in CS or FS cell and mitochondrial concentrations, suggesting a potential role for CS and FS as protective against oxidative insult particularly because of their stability, which does not show a timedependent modification, transformation or degradation.

The literature concerning CS and FS intake from the diet and impact in vivo is limited and almost always in relation to atherosclerotic CVD. Evidence supporting the involvement of environmental factors including diet in the incidence of CVD in the Far East is significantly less than in the West. Genetic reasons for this phenomenon may exist although the rate of this disease in ethnic immigrant populations in the West approaches that of the disease in native Caucasians ${ }^{(47)}$. Konnyaku and Japanese barnyard millet are both widely used as a traditional food all over Japan, the latter especially in cold districts because of its resistance to low temperatures and because its highly nutritious grains make it a good substitute for rice ${ }^{(12,13)}$. Using a cell-based approach, the present study suggests a serotonin derivative-related mechanism against HG-inducing apoptosis in PC12 cells, due to antioxidant properties and ROS-scavenging abilities, in particular superoxide produced in mitochondria. Further studies are needed, especially in vivo, to elucidate other properties and the mechanism of action, which could better clarify the role of serotonin derivatives in this pathology. The present results suggest that these compounds could be taken into consideration to reduce risk against ROS-related neuronal dysfunctions.

\section{Acknowledgements}

The authors are employees of the Kyoto Prefectural University of Medicine and the work described in the present paper has been funded by a Grant-in-Aid for Scientific Research (15390178 TY) from the Ministry of Education, Culture,
Sports, Science and Technology of Japan, from the Biooriented Technology Research Advancement Institution and from the Ministry of Agriculture, Forestry and Fisheries of Japan.

The authors are grateful to Professor Etsuo Niki, Professor Yoshiro Saito and Professor Yasukazu Yoshida, Human Stress Signal Research Centre, AIST Kansai, Japan, for their assistance on HPLC analysis and critical discussion of data.

All the authors designed the proposed study. R. P. conducted the experiments and drafted the paper. Y. N., S. K., O. H. and T. Y. provided helpful technical advice and support in all the experiments carried out, and also supervised the entire work.

The authors are employees of the Kyoto Prefectural University of Medicine. They declare no other conflicts of interest.

\section{References}

1. Dyck PJ \& Thomas PK (editors) (1999) Diabetic Neuropathy, 2nd ed. Philadelphia, PA: WB Saunders.

2. Greene DA, Sima AAF, Alberts JW, et al. (1993) Diabetes mellitus. Theory and practice. Diabetic Neuropathy, pp. 710-755 [H Rifkin and D Porte, editors]. New York: Elsevier.

3. Holder M, Holl RW, Bartz J, et al. (1997) Influence of longterm glycemic control on the development of cardiac autonomic neuropathy in pediatric patients with type I diabetes. Diabetes Care 20, 1042-1043.

4. Phelan SA, Ito M \& Loeken MR (1997) Neural tube defects in embryos of diabetic mice: role of the Pax-3 gene and apoptosis. Diabetes 46, 1189-1197.

5. Szeto HH (2006) Mitochondria-targeted peptide antioxidants: novel neuroprotective agents. AAPS J 8, E521-E531.

6. Fukushima S, Sakata T, Tagawa Y, et al. (1997) Different modifying response of butylated hydroxyanisole, butylated hydroxytoluene, and other antioxidants in $N, N$-dibutylnitrosamine esophagus and forestomach carcinogenesis of rats. Cancer Res 47, 2113-2116.

7. Marchioli R, Schweiger C, Levantesi G, et al. (2001) Antioxidant vitamins and prevention of cardiovascular disease: epidemiological and clinical trial data. Review. Lipids 36, S53-S63.

8. Koyama N, Kuribayashi K, Seki T, et al. (2006) Serotonin derivatives, major safflower (Carthamus tinctorius L.) seed antioxidants, inhibit low-density lipoprotein (LDL) oxidation and atherosclerosis in apolipoprotein E-deficient mice. J Agric Food Chem 54, 4970-4976.

9. Zhang HL, Nagatsu A, Watanabe T, et al. (1997) Antioxidative compounds isolated from safflower (Carthamus tinctorius L.) oil cake. Chem Pharm Bull (Tokyo) 45, 1910-1914.

10. Ly D, Kang K, Choi JY, et al. (2008) HPLC analysis of serotonin, tryptamine, tyramine, and the hydroxycinnamic acid amides of serotonin and tyramine in food vegetables. J Med Food 11, 385-389.

11. Piga R, Naito Y, Kokura S, et al. (2009) Inhibitory effect of serotonin derivatives on high glucose-induced adhesion and migration of monocytes on human aortic endothelial cells. $\mathrm{Br} J$ Nutr 102, 264-272.

12. Niwa T, Murakami K, Ohtake T, et al. (2002) Peroxynitrite scavenging activities of aromatic compounds isolated from Konnyaku, Amorphophallus konjac K.Koch. Biosci Biotechnol Biochem 66, 1386-1388.

13. Watanabe M (1999) Antioxidative phenolic compounds from Japanese barnyard millet (Echinochloa utilis) grains. J Agric Food Chem 47, 4500-4505. 
14. Kumarasamy Y, Middleton M, Reid RG, et al. (2003) Biological activity of serotonin conjugates from the seeds of Centaurea nigra. Fitoterapia 74, 609-612.

15. Sarker SD, Laird A, Nahar L, et al. (2001) Indole alkaloids from the seeds of Centaurea cyanus (Asteraceae). Phytochemistry 57, 1273-1276.

16. Sarker SD, Savchenko T, Whiting P, et al. (1997) Moschamine, cis-moschamine, moschamindole and moschamindolol: four novel indole alkaloids from Centaurea moschata. Nat Prod Lett 9, 189-191.

17. Choi SW, Lee SK, Kim EO, et al. (2007) Antioxidant and antimelanogenic activities of polyamine conjugates from corn bran and related hydroxycinnamic acids. J Agric Food Chem 55, 3920-3925.

18. Cho SH, Lee HR, Kim TH, et al. (2004) Effects of defatted safflower seed extract and phenolic compounds in diet on plasma and liver lipid in ovariectomized rats fed highcholesterol diets. J Nutr Sci Vitaminol 50, 32-37.

19. Kawashima S, Hayashi M, Takii T, et al. (1998) Serotonin derivative, $N$-( $p$-coumaroyl) serotonin, inhibits the production of TNF- $\alpha$, IL- $1 \alpha$, IL- $1 \beta$, and IL- 6 by endotoxin-stimulated human blood monocytes. J Interferon Cytokine Res 18, 423-428.

20. Piga R, Naito Y, Kokura S, et al. (2007) Short-term high glucose exposure induces monocyte-endothelial cells adhesion and transmigration by increasing VCAM-1 and MCP-1 expression in human aortic endothelial cells. Atherosclerosis 193, 328-334.

21. Koyama N, Suzuki K, Furukawa Y, et al. (2009) Effects of safflower seed extract supplementation on oxidation and cardiovascular risk markers in healthy human volunteers. $\mathrm{Br} \mathrm{J}$ Nutr 101, 568-575.

22. Ceriello A (2003) New insights on oxidative stress and diabetic complications may lead to a 'causal' antioxidant therapy. Diabetes Care 26, 1589-1596.

23. Lin MT \& Beal MF (2006) Mitochondrial dysfunction and oxidative stress in neurodegenerative diseases. Nature $\mathbf{4 4 3}$, 787-795.

24. Greene LA (1978) Nerve growth factor prevents the death and stimulates the neuronal differentiation of clonal PC12 pheochromocytoma cells in serum-free medium. J Cell Biol 78, $747-755$.

25. Greene LA, Connolly JL, Viscarello RR, et al. (1979) Rapid, sequential changes in surface morphology of PC12 pheochromocytoma cells in response to nerve growth factor. $J$ Cell Biol 82, 820-827.

26. Grohovaz F, Zacchetti D, Clementi E, et al. (1991) $\left[\mathrm{Ca}^{2+}\right] \mathrm{i}$ imaging in PC12 cells: multiple response patterns to receptor activation reveal new aspects of transmembrane signaling. J Cell Biol 113, 1341-1350.

27. Piga R, Micheletto R \& Kawakami Y (2005) Nano-probing of the membrane dynamics of rat pheochromocytoma by near-field optics. Biophys Chem 117, 141-146.

28. Piga R, Micheletto R \& Kawakami Y (2007) Acoustical nanometre-scale vibrations of live cells detected by a near-field optical setup. Opt Express 15, 5589-5594.

29. Waki A, Yano R, Yoshimoto M, et al. (2001) Dynamic changes in glucose metabolism accompanying the expression of the neural phenotype after differentiation in PC12 cells. Brain Res 894, 88-94.

30. Koshimura K, Tanaka J, Murakami Y, et al. (2002) Involvement of nitric oxide in glucose toxicity on differentiated PC12 cells: prevention of glucose toxicity by tetrahydrobiopterin, a cofactor for nitric oxide synthase. Neurosci Res 43, 31-38.

31. Zou C, Wang Y \& Shen Z (2005) 2-NBDG as a fluorescent indicator for direct glucose uptake measurement. $J$ Biochem Biophys Methods 64, 207-215.

32. Denizot F \& Lang R (1986) Rapid colorimetric assay for cell growth and survival. Modifications to the tetrazolium dye procedure giving improved sensitivity and reliability. J Immunol Methods 89, 271-277.

33. Pigault C, Follenius-Wund A, Schmutz M, et al. (1994) Formation of two-dimensional arrays of annexin $\mathrm{V}$ on phosphatidylserine-containing liposomes. J Mol Biol 236, 199-208.

34. Casciola-Rosen L, Nicholson DW, Chong T, et al. (1996) Apopain/CPP32 cleaves proteins that are essential for cellular repair: a fundamental principle of apoptotic death. J Exp Med 183, 1957-1964.

35. Takahashi M, Shibata M \& Niki E (2001) Estimation of lipid peroxidation of live cells using a fluorescent probe, diphenyl1-pyrenylphosphine. Free Radic Biol Med 31, 164-174.

36. Yoshida Y, Hayakawa M, Habuchi Y, et al. (2006) Evaluation of the dietary effects of coenzyme $\mathrm{Q}$ in vivo by the oxidative stress marker, hydroxyoctadecadienoic acid and its stereoisomer ratio. Biochim Biophys Acta 1760, 1558-1568.

37. Patel KM, Crisostomo P, Lahm T, et al. (2007) Mesenchymal stem cells attenuate hypoxic pulmonary vasoconstriction by a paracrine mechanism. J Surg Res 143, 281-285.

38. Kaliora AC, Dedoussis GV \& Schmidt H (2006) Dietary antioxidants in preventing atherogenesis. Review. Atherosclerosis 187, $1-17$.

39. Liu L, Zubik L, Collins FW, et al. (2004) The antiatherogenic potential of oat phenolic compounds. Atherosclerosis 175, $39-49$.

40. Tanno K, Ichiyanagi T, Ikeshiro Y, et al. (2007) Pharmacokinetic behavior of serotonin derivatives, major antioxidants in safflower seed. J Clin Biochem Nutr 41, Suppl., 94-96.

41. Galati G, Sabzevari O, Wilson JX, et al. (2002) Prooxidant activity and cellular effects of the phenoxyl radicals of dietary flavonoids and other polyphenolics. Toxicology 177, 91-104.

42. Kojima M \& Takeuchi W (1989) Detection and characterization of $p$-coumaric acid hydroxylase in mung bean, Vigna mungo, seedlings. J Biochem 105, 265-270.

43. Mancuso C, Scapagini G, Currò D, et al. (2007) Mitochondrial dysfunction, free radical generation and cellular stress response in neurodegenerative disorders. Front Biosci 12, 1107-1123.

44. Yogeeta SK, Raghavendran HR, Gnanapragasam A, et al. (2006) Ferulic acid with ascorbic acid synergistically extenuates the mitochondrial dysfunction during $\beta$-adrenergic catecholamine induced cardiotoxicity in rats. Chem Biol Interact 163, $160-169$.

45. Oliveira CR \& Rego AC (2003) Mitochondrial dysfunction and reactive oxygen species in excitotoxicity and apoptosis: implications for the pathogenesis of neurodegenerative diseases. Neurochem Res 28, 1563-1574.

46. Ceriello A (2000) The post-prandial state and cardiovascular disease: relevance to diabetes mellitus. Review. Diabetes Metab Res Rev 16, 125-132.

47. Tunstall-Pedoe H, Kuulasmaa K, Amouyel P, et al. (1994) Myocardial infarction and coronary deaths in the World Health Organization MONICA Project. Registration procedures, event rates, and case-fatality rates in 38 populations from 21 countries in four continents. Circulation 90, 583-612. 